\title{
Dp2-Induced Autoantigen/Autoantibody Production From Patients With Systemic Lupus Erythematosus Is Done Through AIM2 And Not NLRP3
}

\author{
Jaw-Ji TSAI ${ }^{1,2,3,4,5^{*}}$ \\ ${ }^{1}$ Center for Translational Medicine, Department of Medical Research, Taichung Veterans General \\ Hospital, Taichung, Taiwan. \\ ${ }^{2}$ Division of Allergy, Immunology and Rheumatology, Department of Internal Medicine, Taichung \\ Veterans General Hospital, Taichung, Taiwan. \\ ${ }^{3}$ Institute of Biomedical Sciences, National Chung-Hsing University, Taichung, Taiwan. \\ ${ }_{4}^{4}$ Institute of Clinical Medicine, National Yang-Ming University, Taipei, Taiwan. \\ ${ }^{5}$ Section of Allergy, Immunology and Rheumatology, Department of Internal Medicine, Asia \\ University Hospital, Taichung, Taiwan.
}

Received: June 20, 2017; Accepted: July 17, 2017; Published: November 27, 2017

*Corresponding author:Jaw-Ji Tsai, MD, PhD, Section of Allergy, Immunology and Rheumatology, Department of Internal Medicine, Asia University Hospital, Taichung, Taiwan. Tel: +886-4-3706-1688 ext.1878; Fax: +886-4-3706-1673; Email: jawjitsai@gmail.com; D00010@auh.org.tw; jawji@ vghtc.gov.tw

\begin{abstract}
Systemic Lupus Erythematosus (SLE) is a disease characterized by the production of autoantibodies against different autoantigens, including double stranded DNA (dsDNA). AIM2 (Protein absent in melanoma 2) is one of the inflammasome proteins which can directly bind to dsDNA. It is therefore possible to speculate that AIM2 may contribute to the disease development of SLE. B cell lines obtained from patients with Dp-allergic SLE were used to investigate the involvement of AIM2 in autoantibody production. The obtained B cells were then cultured with Dp2 for inflammasome activation. The cells-cultured pellet and supernatant were subsequently collected for the measurement of autoantigens, autoantibodies and inflammatory cytokines. The inflammasome of AIM2 and NLRP3 was measured and correlated with autoantibodies production. The B cells were pretreated with siRNA of AIM2, followed by Dp2 stimulation to confirm the activation of AIM2. The results showed that Dp2 could induce inflammasome activation with an increased expression of both NLRP3 and AIM2, which was associated with the production of TRIM21/PGK1, anti-TRIM21/anti-PGK-1 and IL8/IL-1 $\beta$. In the siRNA AIM2 study, Dp2 induced autoantigen/autoantibody/cytokine production could be down-regulated by siRNA in association with a decreased expression of AIM2. Dp2-induced expression of NLRP3 and AIM2 can both be down-regulated by CPP ECP. In Conclusion: Dp2 induced expression of NLRP3 and AIM2 is associated with autoantigen and autoantibody production from B cells derived from Dp-allergic SLE. These autoantigens/autoantibodies can be downregulated only by siRNA AIM2, and not by NLRP3.
\end{abstract}

\section{Introduction}

Systemic Lupus Erythematosus (SLE) is an autoimmune disease characterized by various systemic organ and tissue damage caused by inflammasome activation. Recently enhanced inflammasome activity in SLE has been previously reported [1]. A broad range of microbial, host and environmental triggers of inflammasome have been reported. There are five receptor proteins which have been confirmed to assemble inflammasomes, and include Non-binding Oligomerization Domain (NOD), Leucine-Rich Repeat (LRR)-containing protein, (NLR) family members NLRP1, NLRP3 and NLRP4, as well as the proteins absent in melanoma 2 (AIM2) and pyrin [2, 3].

AIM2 is a new type of inflammasome which is similar to NLR proteins, and can regulate caspase- 1 activation and Interleukin 1 beta (IL-1 $\beta$ ) production in response to viral, host and bacterial dsDNA, and in response to the dsDNA virus vaccinia [4]. AIM2 appears to recognize dsDNA from a variety of microbial species, including those from mammalian cells.

With the existence of a dedicated cytosolic DNA receptor, AIM2 was postulated on the basis of the observation that both microbial and host DNA induce caspase 1 activation in an ASC-dependent manner, but independently of NLRP3, Toll-like Receptors (TLR) or interferon signaling [5]. A link between AIM2 and several human diseases has been previously established. An increased AIM2 expression is associated with psoriasis, abdominal aortic aneurysm and Systemic Lupus Erythematosus (SLE) [6-8]. In the case of psoriasis, autoinflammation could be linked to AIM2- 
mediated recognition of self-DNA in the cytosol of keratinocytes [9]. Similarly, AIM2 can facilitate the apoptotic DNA-induced SLE via arbitrating macrophage functional maturation [10]. By contrast, reduced AIM2 levels correlate with the development of prostate and colorectal cancer as it has consistently been shown that AIM2-deficient mice are hyper-susceptible to colonic cancer development [11-14]. These reports indicate that in addition to its role in host defence, AIM2 also plays a major role in tumor progression, possibly by sensing self-DNA.

Anti-dsDNA is a biomarker of SLE and the concentration is likely related to the disease activity of SLE. Cytosolic dsDNA can be binded to AIM2 directly to trigger inflammasome activation. Its role in IL- $1 \beta$ production, and the link between AIM2 and related family members of inflammasome in the aetiology of SLE, renders AIM2 an attractive target for intervention in this disease. Moreover, chronic arthritis caused by mammalian DNA which escapes from degradation, has also been shown to be associated with increased levels of IL-1 $\beta$ and IL-18 $[15,16]$.

Our previous study showed that the Group 2 major allergen of Dermatophagoides pteronyssinus (Dp2) can induce inflammasome activation through autoantigen/

Autoantibody production by B cells, and that this autoantigen/ autoantibody production was not down regulated by siRNA NLRP3 $[17,18]$. It will be of importance to investigate other family members of inflammasome, particularly AIM2. In this study both NLRP3 and AIM2 were measured in the B cell culture, and siRNA AIM2 was added to the cell to clarify the linkage between AIM2 and autoantigen/autoantibody production in SLE.

\section{Materials and Methods}

\section{Selection of Patients}

HDM-sensitive SLE patients (mite-specific IgE positive in their serum as measured by using the Pharmacia CAP System, Uppsala, Sweden) were selected from the clinic in the Division of Allergy, Immunology and Rheumatology of Taichung Veterans General Hospital. Diagnosis of SLE was made according to the 1997 America College of Rheumatology revised classification criteria for SLE. This study was supported by Taichung Veterans General Hospital (IRB TCVGH No. CE14019A and CE16110B).

\section{Cell Culture}

For the purpose of cell separation and culture, 16-ml blood samples were collected, and the PBMCs were separated by density centrifugation using the Ficoll-Paque Plus density gradient (Pharmacia Biotech, Freiburg, Germany) [15]. Purified $\mathrm{B}$ cells from the PBMCs were prepared as previously described [7]. The B cell preparations were shown to be $95 \%$ positive for the CD19 marker as determined by FACS analysis. The cells were maintained in a RPMI-1640 medium containing inactivated FBS $(10 \%)$, along with $1 \%$ streptomycin/penicillin in a humidified $5 \% \mathrm{CO}_{2}$ atmosphere.

\section{Peptide Synthesis}

CPP ECP (NYRWRCKNQN, 1381Da) was synthesized at Angene
Biotech Co., Ltd., Taiwan, and its purity ( $>90 \%$ ) was assessed through analytical high-performance liquid chromatography. Peptide sequences were confirmed using matrix-assisted laser desorption/ionization, time-of-flight mass spectrometry at Angene Biotech Co., Ltd., Taiwan.

\section{Dp 2 Preparation}

Purified recombinant protein Dp 2 (RP-DP2C-1) was purchased from Indoor Biotechnologies (Charlottesville, Virginia, USA).

\section{Enzyme-Linked Immunosorbent Assay (ELISA)}

Commercially available ELISA kits were used to determine both cytokine and autoantibody levels in the cell culture supernatants. The cell supernatants were collected after Dp2 or CPP ECP treatment during the indicated times, and the levels of IL-1ß'IL-8'IL-6' anti-PGK-1 and anti-TRIM-21 were quantified by ELISA. Plates were read on a SpectraMax M2 microplate reader (Molecular Devices, CA, USA), and analyzed with SoftMax analysis software (Molecular Devices, CA, USA). The means of triplicate ELISA values for each of the dose relationships among these protein expressions were calculated through the use of linear regression.

\section{Western Blotting}

Whole cell lysates were prepared as previously described [18]. After blocking, the blots were incubated with antibodies for anti-human AIM2'NLRP3'MD2'PGK-1'TIM'Enolase-1'TRIM-21 and $\beta$-actin, (Cell Signaling, Massachusetts, USA; Santa Cruz Biotechnology; Millipore, Massachusetts, USA) in TBS overnight at $4^{\circ} \mathrm{C}$, using $0.1 \%$ Tween 20 , followed by three 10 -minute washes in TBS with $0.1 \%$ Tween 20 . The membranes were then incubated using horseradish peroxidase-conjugated, secondary antibodies (Millipore, Massachusetts and USA) for one hour. Detection was performed with ECL (Millipore, Massachusetts, USA), and chemiluminescence was detected by LAS 3000. Band intensity was analyzed by Multi Gauge software V 3.0.

\section{Statistical analysis}

Statistical analyses were performed using graphpad Prism 5 (graphpad Software, San Diego, CA, USA). Data is presented as mean \pm Standard Error of the Mean (SEM). P-values $\leq 0.05$ were considered statistically significant. The pair student's t-test was used to determine the statistical differences between the groups.

\section{Results}

\section{Dp2-Induced Cytosolic Protein Expression from B Cells}

Dp2 induced cytosolic receptor protein production of AIM2, NLRP3 and MD2 from B cells were investigated. B cells derived from patients with Dp-allergic SLE were incubated with Dp2, followed by the measuring of cytosolic receptor protein expression. The results showed that all three cytosolic protein expressions had increased after Dp2 stimulation. Similar results were obtained through LPS stimulation [Figure 1]. 


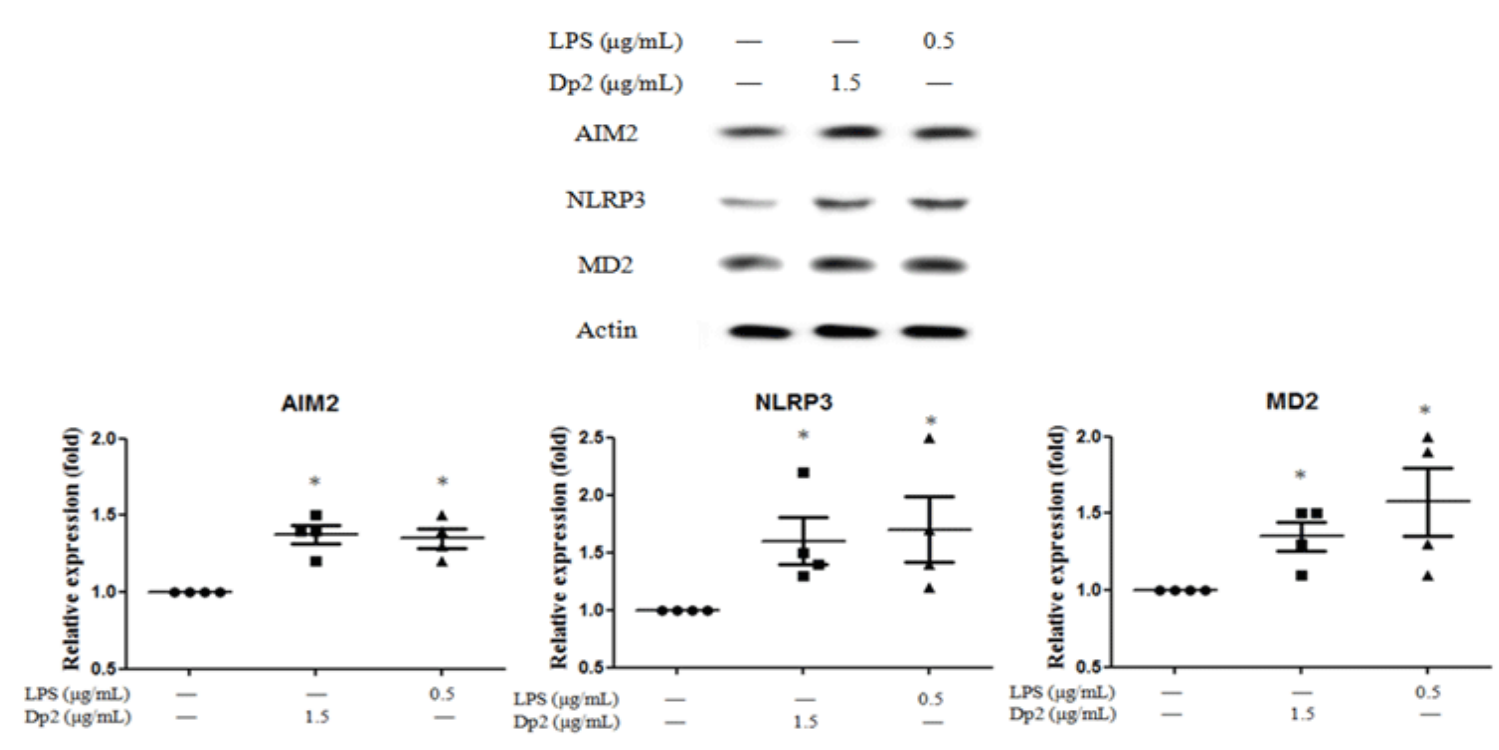

Figure 1: Effect of Dp2-induced cytosolic protein expression from the B cell line.

B cells derived from patients with Dp-allergic SLE $(n=4)$ were incubated with Dp2 for 5 days in a $\mathrm{CO}_{2}$ incubator. Cell pellets were collected for the AIM2, NLRP3 and MD2 measurement. ${ }^{*}$ p $<0.05$ in comparison with buffer control.

\section{Effect of SiRNA AIM2 on the Dp2-Induced Autoantigen Production from B Cells}

The B cells derived from patients diagnosed with Dp-allergic SLE were pre-treated with or without siRNA AIM2, followed by the stimulation of Dp2. Cell pellets were collected for obtaining the measurements of autoantigens. The results showed that AIM2 expression could be down regulated through the use of siRNA AIM2 (n=4). Dp2 induced autoantigen production of both PGK-1 and TRIM-21 from the B cells could be down-regulated by siRNA AIM2, with no effect on the expression on TIM and Enolase-1 in comparison with buffer control [Figure 2].

\section{Effect of SiRNA AIM2 on the Dp2-Induced Autoantibody} and Cytokine Production from B Cells

B cells derived from patients with Dp-allergic SLE were incubated with or without siRNA AIM2, followed by the stimulation of Dp2. Cell cultured supernatant was collected for obtaining the measurements of autoantibodies and cytokines. The results showed that Dp2-induced production of anti-PGK-1 and anti-TRIM21 could be down regulated by siRNA AIM2 $(n=4)$. Similar results showed that Dp2-induced production of IL-1 $\beta$ and IL-8 could be down regulated by siRNA AIM2 [Figure 3].

\section{Effect of CPP ECP on Dp2-Induced Cytosolic Protein Expression from the $B$ cell Line}

B cells derived from patients with Dp-allergic SLE were incubated with Dp2 with or without CPP ECP. Cell pellets were collected for obtaining the measurements of AIM2, NLRP3 and MD2. The results showed that CPP ECP could down regulate the expression of AIM2 and NLRP3, but not MD2 (n=4) [Table 1].
B cells derived from patients with Dp-allergic SLE $(n=4)$ were incubated with Dp2 with or without CPPecp for 5 days in a CO2 incubator. Cell pellets were collected for the AIM2, NLRP3 and MD2 measurement. ${ }^{*} \mathrm{p}<0.05$ in comparison with buffer control. $\# \mathrm{p}<0.05$ in comparison with Dp2.

Table 1: Effect of CPPecp on Dp2-induced cytosolic protein expression from the B cell line.

\begin{tabular}{|c|c|c|c|c|} 
& Control & Dp2 & Dp2+CPPecp & LPS \\
\cline { 2 - 5 } & Mean \pm SEM & Mean \pm SEM & Mean \pm SEM & Mean \pm SEM \\
\hline AIM2 & $1.0 \pm 0.0$ & $1.7 \pm 0.3^{*}$ & $1.2 \pm 0.1 \#$ & $1.8 \pm 0.6^{*}$ \\
\hline NLRP3 & $1.0 \pm 0.0$ & $1.7 \pm 0.4^{*}$ & $1.0 \pm 0.3 \#$ & $1.6 \pm 0.5^{*}$ \\
\hline MD2 & $1.0 \pm 0.0$ & $4.3 \pm 2.0$ & $3.2 \pm 1.4$ & $3.9 \pm 1.7$ \\
\hline
\end{tabular}

\section{Discussion}

SLE is a disease characterized by the production of autoantibodies against different autoantigens. Some of these autoantibodies have exhibited a link between titers and disease activity, such as the anti-dsDNA autoantibody. More than $95 \%$ of untreated SLE anti-dsDNA could possibly be detected in the sera. It is believed that SLE disease activity can be triggered by various environmental factors including sex hormones, ultraviolet light, infections and certain medications and chemicals. Our previous reports have shown that Dp can trigger autoantigen/ autoantibody production from B cells derived from patients with Dp allergic SLE through bystandard activation $[15,16]$. There are five receptor proteins which have been confirmed to assemble in inflammasome, including NLRPs and AIM2. In this study both NLRP3 and AIM2 could be up-regulated by Dp2 stimulation, suggesting that both inflammasome proteins can be induced by Dp2. 





TIM

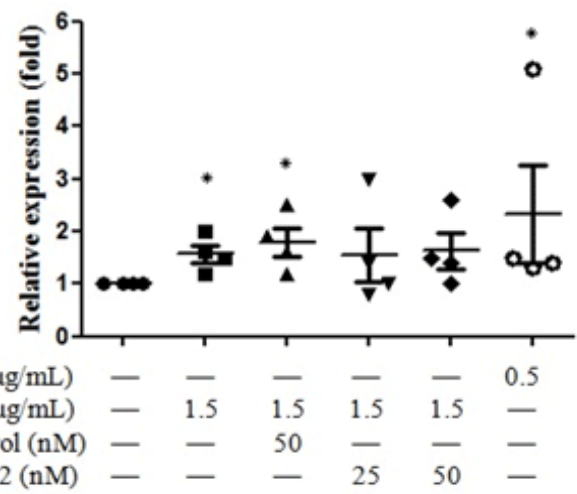

TRIM-21



Enolase-1



Figure 2: Effect of siRNA AIM2 on the Dp2-induced autoantigen production from B cells.

B cells $(n=4)$ derived from patients with Dp-allergic SLE were pre-treated with or without siRNA AIM2 for 3 days, followed by the stimulation of Dp2 for 5 days in the $\mathrm{CO}_{2}$ incubator. Cell pellets were collected for the measurement of autoantigen. ${ }^{*} \mathrm{p}<0.05$ in comparison with buffer control. \#p $<0.05$ in comparison with Dp2 


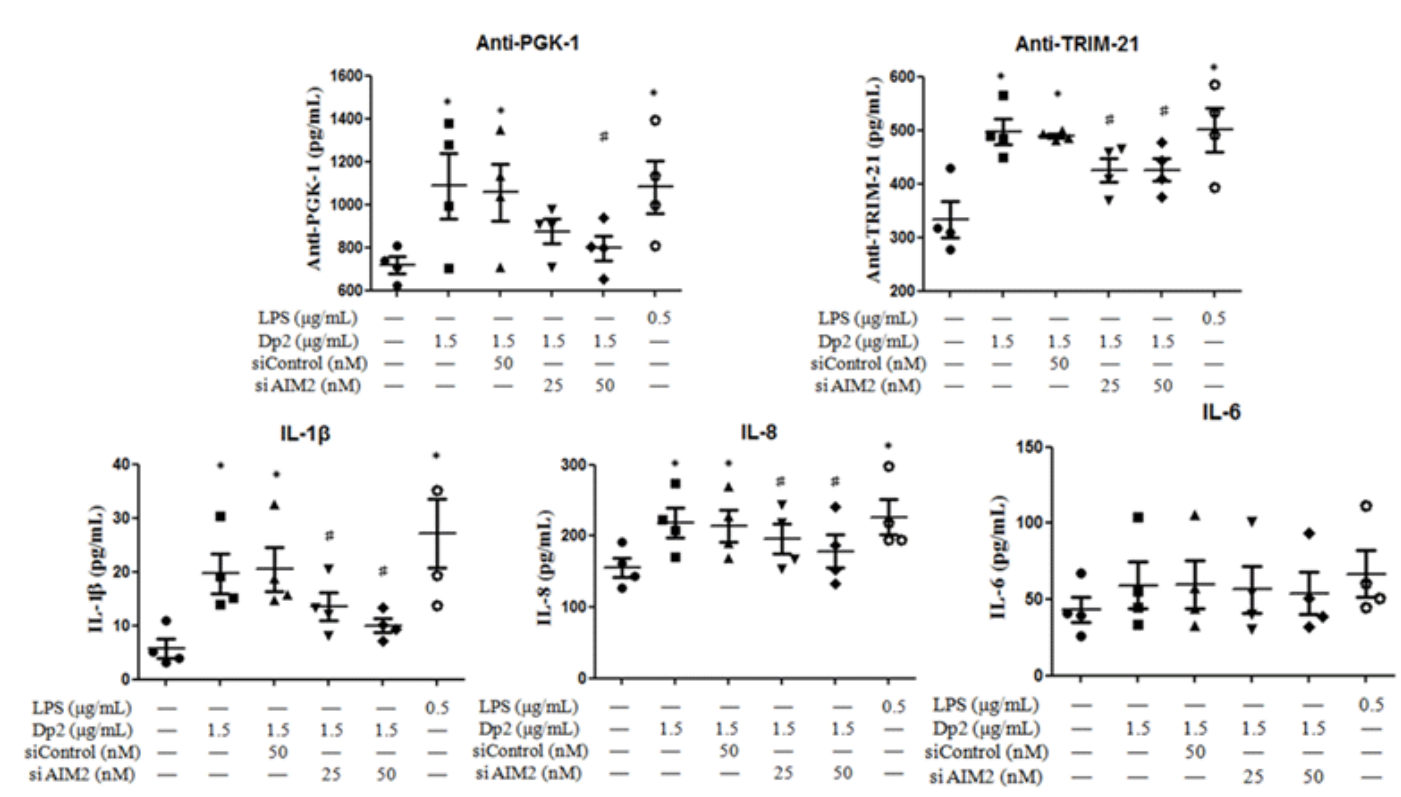

Figure 3: Effect of siRNA AIM2 on the Dp2-induced autoantibody and cytokine production from B cells.

B cells $(n=4)$ derived from Dp-allergic SLE were incubated with or without siRNA AIM2 for 3 days, followed by the stimulation of Dp2 for 5 days in the $\mathrm{CO}_{2}$ incubator. Cell cultured supernatant was collected for the measurement of autoantibodies and cytokines.

Since AIM2 is a sensor of cytoplasmic DNA, it can bind dsDNA directly and is considered to be relevant to the regulation of autoimmune response in SLE. In this Dp2-induced autoimmune response, the assembling of AIM2 could be inhibited through the pretreatment of its siRNA, while the decreased assembling of AIM2 has been correlated with the down-regulation of autoantigen/autoantibody production. These results suggest that the activation and assembling of AIM2 may play at least in part, a role in the pathogenesis of autoimmune in SLE. The results show that both AIM2 and NLRP3 could be down-regulated by CPP ECP. These results suggest that CPP ECP is non-specific in immuno modulation with regards to the Dp2-induced immune response.

A previous study has shown that Dp2 can induce autoantigen/ autoantibody production in Dp-allergic SLE patients, but our patient's autoantigen/autoantibody production was not downregulated due to the pre-treatment of siRNA NLRP3 [18]. In the present study, Dp2-induced inflammation activation to generate autoantigens/autoantibodies is associated with the assembling of NLRP3 and AIM2 proteins. However, only AIM2 assembling is for autoantigen/autoantibody production, since this autoantigen/ autoantibody production can be down-regulated by the successful inhibition of AIM2 through siRNA AIM2. These results suggest that a biomarker for AIM2 is specific autoantigen/autoantibody production. The major limitation of this study was that there were too few patients enrolled, and no figures on decrease in activity were collected to correlate with the expression of AIM2. This cannot be clarified until more cases are included. Whether AIM2 can be used as a biomarker for SLE disease activity still requires further investigation. In conclusion, Dp2-induced autoantigen/ autoantibody production from B cells derived from Dp-sensitive SLE patients occurs through AIM2, and not NLRP3.

\section{References}

1. Liu J, Berthier CC, Kahlenberg JM. Enhanced Inflammasome Activity in Systemic Lupus Erythematosus is Mediated via Type I Interferon up Regulation of Interferon Regulatory Factor 1. Arthritis Rheumatol. 2017; 69(9): 1840-1849; Doi: 10.1002/art.40166

2. Von Moltke J, Ayres JS, Kofoed EM, Chavarria-Smith J, Vance RE. Recognition of bacteria by inflammasomes. Annu Rev Immunol. 2013; 31:73-106; Doi: 10.1146/annurev-immunol-032712-095944

3. Broz P, Monack DM. Newly described pattern recognition receptors team up against intracellular pathogens. Nat Rev Immunol. 2013; 13(8):551-565; Doi: 10.1038/nri3479

4. Hornung V, Ablasser A, Charrel-Dennis M, Bauernfeind F, Horvath G, Caffrey DR, et al. AIM2 recognizes cytosolic dsDNA and forms a caspase-1-activating inflammasome with ASC. Nature. 2009 26; 458(7237):514-518; Doi: 10.1038/nature07725

5. Muruve DA, Pétrilli V, Zaiss AK, White LR, Clark SA, Ross PJ, et al. The inflammasome recognizes cytosolic microbial and host DNA and triggers an innate immune response. Nature. 2008; 452(7183):103107; Doi: 10.1038/nature06664

6. Dihlmann S, Erhart P, Mehrabi A, Nickkholgh A, Lasitschka F, Böckler $D$, et al. Increased expression and activation of absent in melanoma 2 inflammasome components in lymphocytic infiltrates of abdominal aortic aneurysms. Mol Med. 2014; 20:230-237; Doi: 10.2119/ molmed.2013.00162

7. Yvonne D, Mark P, Sarah K, Claudia K, Christine G, David A, et al. Cytosolic DNA triggers inflammasome activation in keratinocytes in psoriatic lesions. Sci Transl Med. 2011; 3(82): 82ra38; Doi: 10.1126/ scitranslmed.3002001 
8. Javierre BM, Fernandez AF, Richter J, Al-Shahrour F, MartinSubero JI, Rodriguez-Ubreva J Et al. Changes in the pattern of DNA methylation associate with twin discordance in systemic lupus erythematosus. Genome Res. 2010; 20(2):170-179; Doi: 10.1101/ gr.100289.109

9. Dihlmann S, Tao S, Echterdiek F, Herpel E, Jansen L, Chang-Claude J, et al. Lack of absent in melanoma 2 (AIM2) expression in tumor cells is closely associated with poor survival in colorectal cancer patients. Int J Cancer. 2014; 135(10):2387-2396; Doi: 10.1002/ijc.28891

10. Divaker C. Ravichandran P, Xin D, Hongqi L, Hongzhu L. Emerging Roles for the Interferon-Inducible p200-Family Proteins in Sex Bias in Systemic lupus Erythematosus. J Interferon Cytokine Res. 2011; 31(12): 893-906; Doi: 10.1089/jir.2011.0073

11. Ponomareva L, Liu H, Duan X, Dickerson E, Shen H, Panchanathan R, et al. AIM2, an IFN-inducible cytosolic DNA sensor, in the development of benign prostate hyperplasia and prostate cancer. Mol Cancer Res. 2013; 11(10):1193-1202; Doi: 10.1158/1541-7786.MCR-13-0145

12. Man SM, Zhu Q, Zhu L, Liu Z, Karki R, Malik A, et al. Critical role for the DNA sensor AIM2 in stem cell proliferation and cancer. Cell. 2015; 162(1):45-58; Doi: 10.1016/j.cell.2015.06.001
13. Wilson JE, Petrucelli AS, Chen L, Koblansky AA, Truax AD, Oyama Y, et al. Inflammasome-Independent Role Of AIM2 In Suppressing Colon Tumorigenesis Via DNA-PK And Akt. Nat Med. 2015; 21(8):906-913; Doi: $10.1038 / \mathrm{nm} .3908$

14. Kawane K, Ohtani M, Miwa K, Kizawa T, Kanbara Y, Yoshioka Y, et al. Chronic polyarthritis caused by mammalian DNA that escapes from degradation in macrophages. Nature. 2006; 443(7114):998-1002; Doi: $10.1038 /$ nature 05245

15. Nagata S. Autoimmune diseases caused by defects in clearing dead cells and nuclei expelled from erythroid precursors Immunol Rev. 2007; 220:237-250; DOI: 10.1111/j.1600-065X.2007.00571.x

16. Wu CC, Liao EC, Lee MF, Tsai JJ. Augmentation of regulatory T cells in allergic individuals by recombinant Der $\mathrm{f} 2$ peptide with fungal immunomodulatory peptide fve. Ann Allergy Asthma Immunol. 2009; 102(3):216-222; Doi: 10.1016/S1081-1206(10)60084-1

17. Yu SJ, Liao EC, Tsai JJ. Der p 2 can induce bystander activation of B cells derived from patients with systemic lupus erythematosus. Immunobiology. 2014; 219(12):958-963; Doi: 10.1016/j. imbio.2014.07.018

18. Chi-Xin YE, Sheng-Jie YU, Ching-Yun CHANG, Jaw-Ji TSAI. Dp2-Induce Autoantigen/Autoantibody Production from Patients with Systemic Lupus Erythematosus and Its Modulation by Cppecp. J Rheumatol Arthritic Dis. 2017; 2(1):1-6. 